\title{
A Prototype Automatic Solar Panel Controller (ASPC) with Night-time Hibernation
}

\author{
Salihu O. Aliyu, Michael Okwori and Elizabeth N. Onwuka \\ Telecommunication Engineering Department, Federal University of Technology Minna, Nigeria \\ E-mail: \{salihu.aliyu, michaelokwori, onwukaliz\}@futminna.edu.ng
}

\begin{abstract}
Solar cells, as an alternate means of electricity supply, is rapidly advancing. Generally, output of solar cells depends largely on intensity of sun and angle of incidence on the cells. This means that to get maximum efficiency from these cells, they must remain directly pointed at the sun from sun rise to sun set. However, the position of sun's highest intensity with respect to a given spot changes with time of the day. It is therefore necessary to automatically control position of solar cells to always align with the highest intensity of sun. In this paper, we present a prototype automatic solar panel controller, with night time hibernation. The proposed system consists of both software and hardware parts, and it automatically provides best alignment of solar panel with sun to get maximum intensity. The solar panel controller system detects the presence of sun rays using light dependent resistors (LDR). At the heart of the control mechanism is an AT89C52 microcontroller. It is programmed to constantly monitor the output of an LDR, actuate a stepper motor to reposition the solar panel to a direction with the highest intensity. The proposed system also has an option of manual control of the panel via a computer interface or a keypad unit for easy of user interactivity during maintenance. Testing the proposed system, results shows that it can successfully track the sun and enter idle mode in the absence of sun rays, hence, conserving over $50 \%$ of energy required to operate the system.
\end{abstract}

Index Terms-Automation, solar power, renewable energy, hibernation, sun tracker.

\section{INTRODUCTION}

One of the most common problems facing developing countries today, especially African countries, is the shortage of energy. This proble $m$ is as a result of increase in de mand for electrical energy and high cost of fuel. The authors, like many others, believe that part of the solution to the energy crisis lies in using renewable energy sources such as solar energy, wind energy, etc. Nowadays, solar energy is widely used in our environment, and its use is expected to grow considerably in coming years. Since output of solar panel depends largely on intensity of sun reaching it and the angle of incidence, there is a need to increase efficiency of solar panel by keeping it aligned to the sun at proper angle to its rays, from sunrise till sun set. However, most solar panels, in uset today in African countries, are fixed to a given position, while the earth rotates with respect to the sun. This means that, for a greater part of the daylight period, the solar panel stays away from the position of the suns greatest intensity. To solve this problem, researchers have been on the frontline to see how a system could be designed such that it has the capability of positioning solar panel with respect to the motion of the earth around the sun, without the need for human intervention.

The rest of this paper is organized as follows: in section II, we presented review of related work in the literature. Design and implementation of the proposed system is presented in section III, while section IV discussed results and discussions of the implemented system, and finally, conclusion is drawn in $\mathrm{V}$.

\section{RELATED WORKS}

Among researchers who have made attempt to solve this problem is [1]. They presented design and construction of an automatic sun tracking system which is capable of providing best alignment of solar panel with the sun. In their design, two AT89C52 microcontrollers were used. One of the microcontrollers was used as a master controller to control automatic operation of the tracker, while the other as a slave, communicates serially with the computer interface through an installed Visual Basic (VB) application.

In [2], a multifunctional sun tracking system whose central control unit is based on a microcontroller was presented. They used three LDR sensors to track position of the sun and rotate a stepper motor accordingly. This design has a limitation because, during the absence of sun radiation (i.e., at night times), the solar panel is rotated continuously to meet up with the next sun location. As a result of the continuous rotation of the stepper motor, part of the energy stored in the battery is consumed. In [3], the authors designed and simulated a sun tracking solar power system. The simulation was realized using MATLAB/Simulink platform. The system consists of four modules: solar tracking cells, signal conditioning circuit, controller, and motor. The simulation provides an excellent platform for students to study the different theories involved in designing sun tracker for solar power system.

In [4], the author presented design and construction of a PIC based automatic solar radiation tracker. The system uses PIC $16 F 877$ microcontroller to control the stepper 
motor based on in-circu it current measured from the solar module. The solar panel is rotated to the side which gives higher in-circuit current. It has a unique feature of using the solar module itself as tracking sensors, hence lowering the complexity of the device and its cost. Among other solutions presented by past researchers is a sun tracking system based on time. It tracks the sun according to time of day without taking into account seasonal variations in sun position, and presence of sun radiation [5]. A related solution was provided by [6], however, the system requires a reset button to be pressed before it could resume from sleep mode, therefore requiring human intervention.

In [7], the authors described design and construction of a tracking system which can be used with single-axis solar concentrating systems. Three Light Dependent Resistors (LDR) were used to get the position and status of the Sun. One of the LDR detects whether the collector is focused, a second LDR determines if there is cloud cover, and the third senses whether it is day or night. In their work, a DC motor was used to rotate the solar concentrating system. A similar work on one-axis tracking was presented by [8]. The authors proposed design and application of one-axis sun tracking system which follows position of the sun. It was used to investigate effects of one-axis tracking system on the solar energy in Turkey. In their design, they included a serial communication interface based on RS 485 to monitor whole processes on a computer screen and to plot data as graphics. Other related works were presented by [9]-[11][12]. Some efforts have also been vested on the design of two-axis tracker [13]-[16] to increase the radiation received by the $\mathrm{PV}$, however, the design involves more complexity when compared to single-axis tracker. The authors in [16] presented theoretical works for solar radiation and angle of incidence values of any location, in addition to an experimental study carried out on a system tracking the sun in two axes and in a fixed system. Reviews of sun tracking systems, principle and methods are presented in [17], [18].

In [19], [20], the authors compared the performance of single and dual axis tracker. They concluded that both have better performance than a fixed mount panel. However, dual axis has better efficiency than single axis but with greater design complexity. Similarly, in [21], a sun tracking mechanis $m$ for single and dual axis tracker using Programmable Logic Controller (PLC) was proposed. Their results showed that dual axis can have good efficiency over single axis, though, with additional complexity.

In [22], an optimal PID controller design using swarm intelligence algorithms for sun tracking system was proposed. The authors focused on the control of a DC motor which can be used for dual-axis. In particular, they designed an optimum proportional-integral-derivative (PID) controller for DC motors of dual axis solar tracker system. The problem was formulated as optimization problem and three swarm intelligence based metaheuristic algorithms namely particle swarm optimization (PSO), fire fly algorithm (FFA) and Cuckoo
Search Algorithm (CSA) were used for optimum tuning of the PID controller. The study shows that CSA has faster convergence rate, small variance and standard deviation of design parameters as compared to FFA and PSO. As for performance, FFA and CSA show better performance as compared to PSO for the problem do main under study. In [23], an intelligent control method for the maximum power tracking (MPPT) of a PV system was proposed. The proposed system works under variable temperature and solar irradiation conditions. The authors presented a simulation study of the MPPT system which was carried out using artificial neural network. The proposed system was compared with Perturb and Observe method. The simulation results obtained shows that the ANN approach has good performance response.

The authors in [24] proposed a versatile fuzzy controller which could be capable of effective and promise control of the satellite attitude and sun tracking at the same time in the presence of disturbance torques. The work focused on theoretical design and simulation of the governing equations. In [25], a fuzzy logic based controller was design using FPGA for sun tracking in solar array system. The tracking system composed of a PV module, stepper motor, sensors, and input/output interface etc. The proposed system was simulated using MATLAB/Simulink program and excellent result was obtained. A $24 \%$ energy efficiency than a fixed sun panel system was obtained. In [26] the authors presented a comparative analysis of fixed and sun tracking low power PV systems putting energy consumption into consideration. The experimental results obtained from their work highlight the significant increase $12-20 \%$ of the produced energy by using the sun tracker as compared to the fixed PV system. In addition, the results obtained during "clear sky" days show that any solar tracker must be designed such that to limit the energy consumption caused by small variations of solar irradiation. The authors further suggested that trackers need to be designed such that the energy consumption as sociated with unneces sary rotations of PV source can be limited.

In this paper, we proposed design and implementation of a system capable of providing best align ment of solar panel with the sun. The proposed system tracks the sun radiation only when it is available, otherwise, it hibernates to save energy. Other features include emergency control of the panel via a computer interface with an installed software, keypad interface for standalone application (i.e. no computer interface). The tracking status of the system is also displayed on an LCD (liquid crystal display) and/or through the computer interface. Our proposed system has more function as compared to other works proposed in the literature.

A single axis tracker has one degree of freedom which could be vertical or horizontal. Based on this, several implementations of single axis trackers have been proposed. A typical horizontal single axis tracker is shown in Fig. 1. The mechanism of a single axis tracker only rotates in one plane around a single axis from east to west. It offers lower cost and higher reliability since it is less complex as compared to dual axis tracker. 


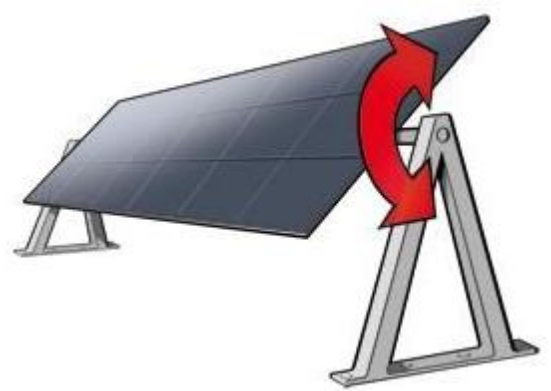

Fig.1. Horizontal single axis tracker, [27]

\section{DESIGN AND IMPLEMENT ATION}

In designing our proposed system, care was taken to ensure all required components available locally at considerably low price. We considered using Atmel 1 AT8C52 microcontroller which is the cheapest among available microcontrollers in our region. This version was selected, as compared to other 8052 family, because it has the necessary features required by our system. To rotate the panel, stepper motor was used because it can be controlled in steps and no feedback circuit is required to know its current position. A single chip current driver IC was used to drive the stepper motor. The entire system was designed to be user friendly with visual interface from PC, including LCD display and manual control unit for troubleshooting and emergency control.

\section{A. Hardware Implementation}

The block diagram of the proposed system is as shown in Fig. 2. It consists of the following units: sensing, PC, keypad interface, and motor driver units etc.

The sensor unit comprises of three LDRs to sense the intensity of the sun as compared to six LDRs used in [1] Resistance of LDR is inversely proportional to intensity of light falling on it. In this design, the LDR is connected in series with a variable resistor to form a voltage divider network, and the output voltage is taken across the variable resistor as shown in Fig. 3. Similar circuits to Fig. 3 were designed for the three Sun Tracking Sensors (STS1, STS2, and STS3).

As the light intensity falling on LDR increases, the output voltage increases and vice versa. The output voltage was used to trigger a NE555 timer connected in mono-stable mode. Whenever the LDR is in darkness/shadow, the output of the timer is held HIGH, while it is held LOW if LDR is under rays of light. Figs. 4 and 5 show sensor location before and after panel rotation. The third sensor was placed separate from the two tracking sensors. Whenever the two tracking sensors are in darkness/shadow for a given period of time, (we programmed our system to use two minutes) the system enters idle mode. The next appearance of the sun is detected by the third sensor, which sends an interrupt signal to the microcontroller. The interrupt signal toggles the mic rocontroller automatically back to tracking mode

\footnotetext{
${ }^{1}$ http://www.atmel.com/
}

without any human intervention, as also in [19]. The keypad unit provides an option for manual control of the solar panel, which could either be an anticlockwise or clockwise rotation. The PC interface also provides a similar control of the panel as the keypad unit and displays the current status of the tracking system, similarly as in [28]. We used stepper motor to drive the solar panel.

A stepper motor is an electromechanical device, which converts electrical pulses into discrete mechanical movements. The shaft of the stepper motor rotates in discrete step increments when electrical command pulses are applied to it in the proper sequence [29]. Stepper motor was selected for this design due to its ability of being controlled accurately in an open loop system. This type of control eliminates the need for expensive sensing and feedback device such as optical encoder [29]. The two common types of stepper motors are unipolar and bipolar stepper motor. This system was design to use unipolar stepper motor. There are three different modes of rotating stepper motor. These are: single coil excitation, double coil excitation, and half step excitation. We have used single coil excitation in this design. In single coil excitation, a coil is energized at a time. To make a complete rotation, the coils are energized one at a time in the proper sequence.

\section{B. Software Implementation}

The software programs written for the proposed system consist of two parts; one for the AT89C52 microcontroller and the other for the PC interface. We discuss these two parts in this section.

Microcontroller is a programmable chip, which needs to be pre-programmed before it can be used in any application. In this work, it was programmed to provide automatic control function for the solar panel. It has numerous robust features such as low power consumption, good speed of operation and ease of developing application with it [30], [31]. In this design, only one AT89C52 was used as compared to [1]. AT89C52 microcontroller was used due to its availability in a wide array of variations from manufacturers such as Intel $^{2}$, Philips ${ }^{3}$, Dallas ${ }^{4}$, and Siemens ${ }^{5}$, especially for the fact that it is low cost and easily available in Nigeria.

The architecture of the AT89C52 provides it with very valuable features [31]. We programmed the chip in Clanguage using $\mathrm{Keil}^{6} \mu$-Vision version 3 editor. It was programmed to monitor and control other peripherals connected to it. It monitors the output of the NE555 timer from the sensor unit to know the direction to rotate the panel. On power on, the system is initialized and the solar panel is set to its in itial position before tracking starts. At the end of the day, the panel is returned to its initial position and the system enters into idle mode until sunrise the next day before it resumes tracking. The third sensor (STS3), detects the presence of sun rays, sends an output

\footnotetext{
${ }^{2}$ http://www.intel.com/en US/worldwide.htm ${ }^{3}$ http://www.philips.com/global/

${ }^{4} \mathrm{http}: / /$ www.dallaselectronics.com/

${ }^{5}$ http://www.siemens.com/entry/cc/en/
} 
signal which serves as an interrupt signal to the microcontroller. On detecting the interrupt, the microcontroller executes the interrupt service routine, which returns the system to tracking mode. The flow chart of the system's operational sequence is as shown in Figs. 6 and 7.

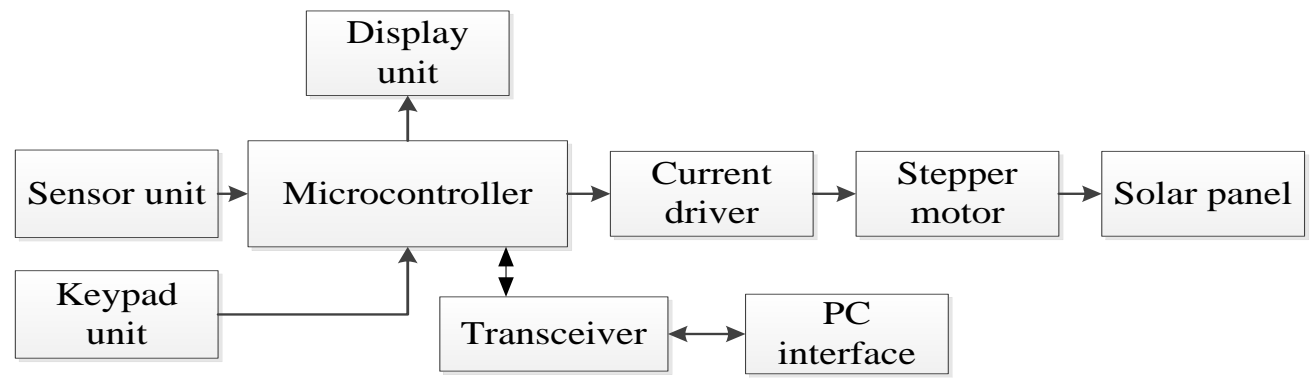

Fig.2. Block Diagram of Automatic Solar Panel Controller

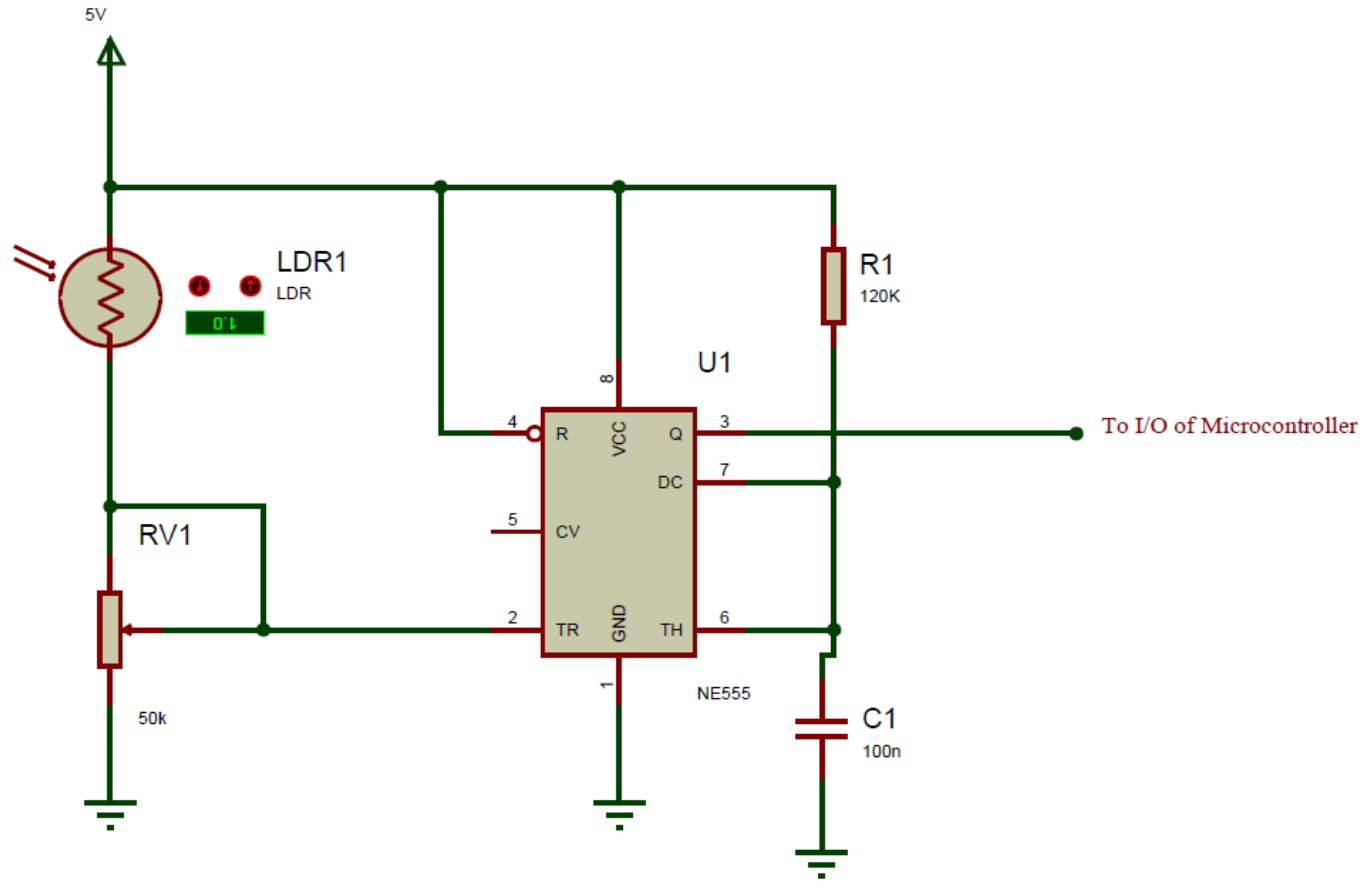

Fig.3. LDR connection to NE555 timer

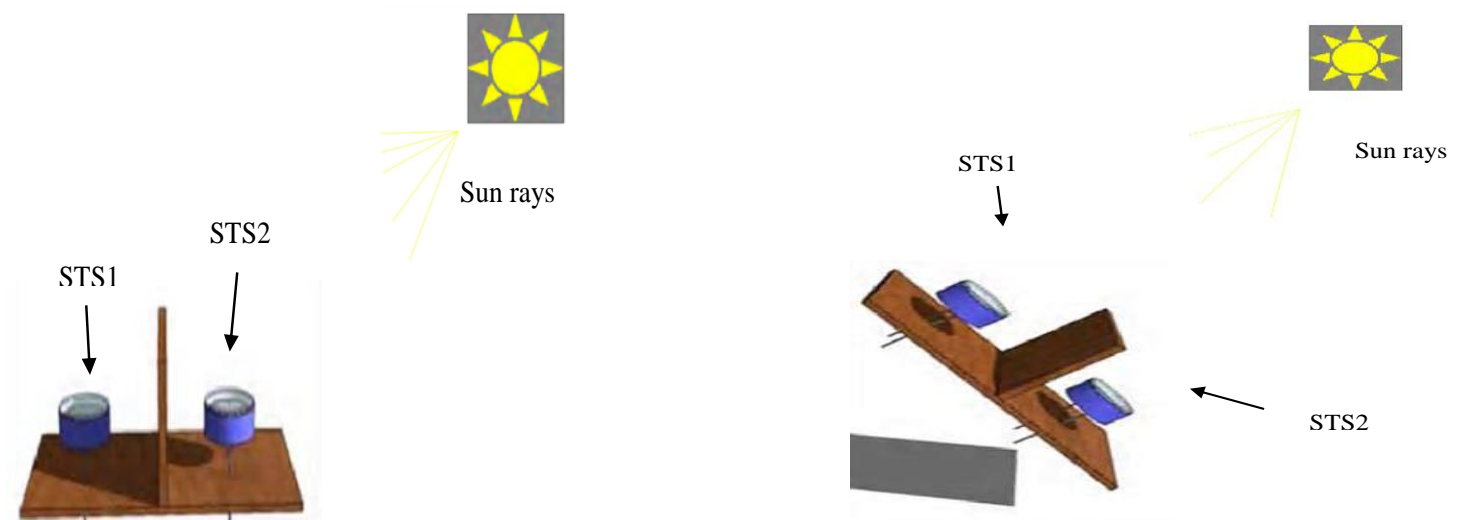

Fig.4. Position of the LDRs before rotation

Fig.5. Position of LDRs after rotation 


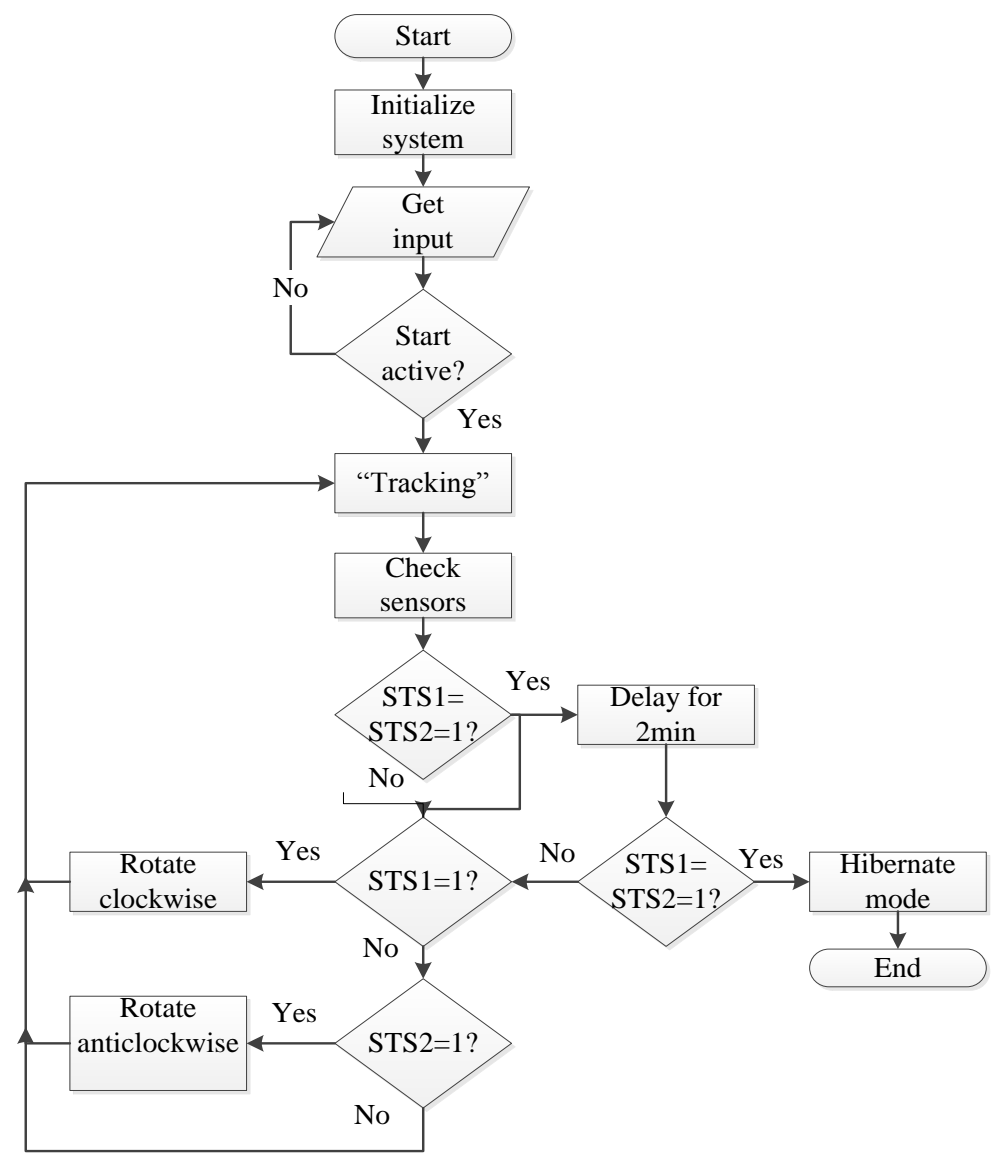

Fig.6. Flowchart of automatic tracking

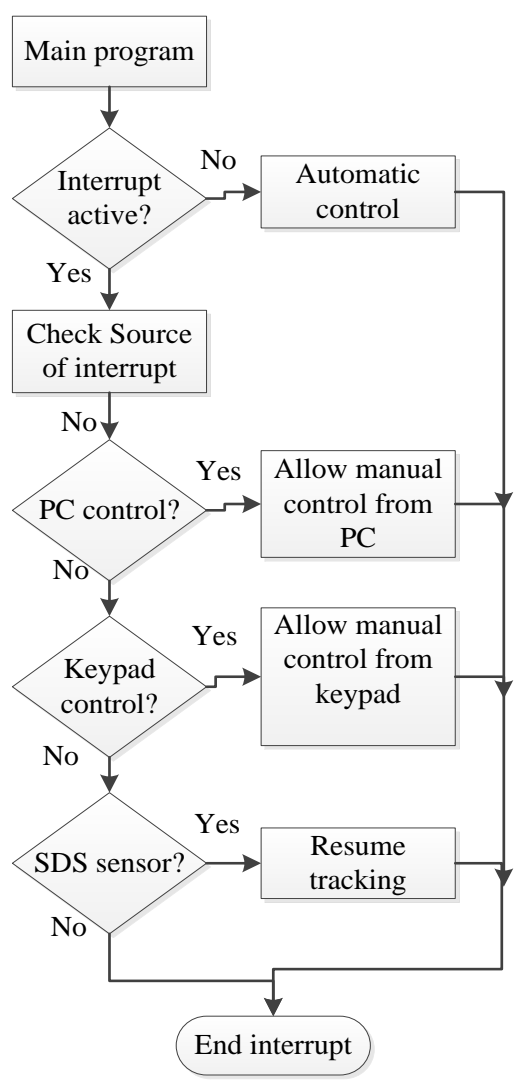

Fig.7. Interrupt operation flowchart

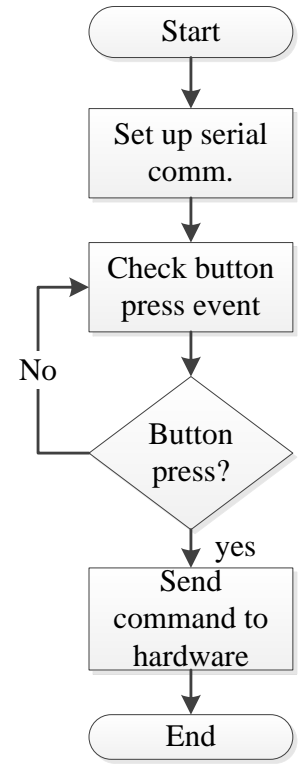

Fig.8. Flowchart for PC interface

The PC control interface program was written using the Microsoft Visual Basic (VB) programming language (version 6). A Graphical User Interface (GUI) was created using VB. The GUI holds the command button and other functions. An RS232 communication link to the hardware unit was created using MSComm. The link was set at the same baud rate as the hardware unit. In particular, our system used the following communication 
settings: $9600 \mathrm{bps}$, with 8bits, 1startbit, 1stopbit and no parity. Figs. 8 and 9 show the flow chart of this unit and the interface respectively.

We implemented control of only "ASPC 1" (i.e. control of one panel), other options as shown in the interface are for future expansion. Therefore the option "ASPC 1" is selected as shown in Fig. 9.

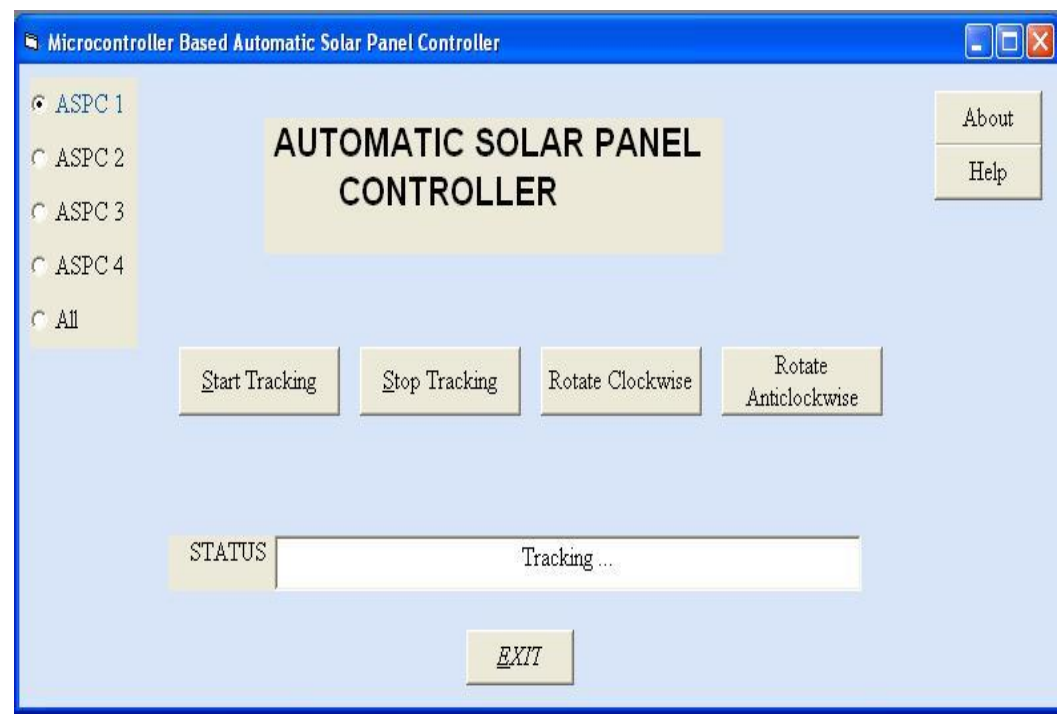

Fig.9. PC control interface

\section{RESULTS AND DISCUSSION}

As mentioned earlier, the tracking software was written in C-language and compiled using the Keil ${ }^{6} \mu$-Vision version 3 simulator. The hexadecimal file was transferred to the microcontroller using TOP2005+ ${ }^{7}$ universal programmer. After construction, we tested the different features of our proposed system as follows: First, we tested the tracking function of the ASPC by varying the intensity of light falling on the LDRs. When the output of STS1 (sun tracking sensor 1) is high, rotation of the panel was observed. This condition indicates to the microcontroller that the sensor is in darkness/shadow. Then the microcontroller sends signal to the motor driving unit to rotate the panel to opposite direction of STS1. Similar test was carried out on STS2, i.e., creating darkness/shadow around it. It was also observed that the panel rotates to the direction of light rays. In this manner, tracking is continued as long as there are light rays.

Secondly, hibernation feature was tested. In the absence of light rays, after the programmed time, the system was observed to enter idle mode to save energy. In particular, 2 minutes was used considering our location. Experimentally, it was observed that the length of shadow covers our sensors in every two minutes. Therefore two minutes was set as the duration of delay for checking the condition of the sensors.

Likewise at night, similar result was observed. Tracking was observed to resume immediately light rays was made available. This was achieved through the SDS (sun detecting sensor), i.e. STS 3, which generates an

\footnotetext{
${ }^{6} \mathrm{http}: / / \mathrm{www}$. keil.com/

${ }^{7}$ http://www.top2048.com/
}

interrupt signal whenever it detects light rays. With this, the system achieved tracking when the sun radiation is available and stops when it is absent without the need for human intervention. Next, manual control of the system was tested using the provided keypad unit. To activate manual control, an emergency push button is first pressed. Then, we observed the rotation of the panel whenever "Rotate clockwise" button was pressed. Similarly we observed the case when "Rotate anticlockwise" button was pressed. Other manual control functions include start and stop tracking functions. It was observed that the proposed system responds to all the manual control from the keypad unit.

Finally, we tested the communication link between the PC interface and the tracking system. While, the hardware unit is on, we launched the PC interface software which is connected to the hardware via an RS232 to USB serial cable. The interface also contains similar control functions as the keypad unit. On each button press, the hardware unit was observed to respond to the corresponding command and system status is displayed on the interface as well as the LCD display connected to the hardware. Generally, the different system status are shown on the PC interface as well as the LCD unit. The proposed system can work in standalone mode without the need for the PC interface. Comparing our work to previous literature, tracking can be stopped or resume without the need to press any button as in [6], less numbers of microcontroller was used as compare to [1].

Figure 10 shows the total possible radiation that can be absorbed in 24 hours when the tracker is employed in comparison to a fixed solar panel. This shows the advantage of a solar panel equip with sun tracker over the fixed position solar panel. 


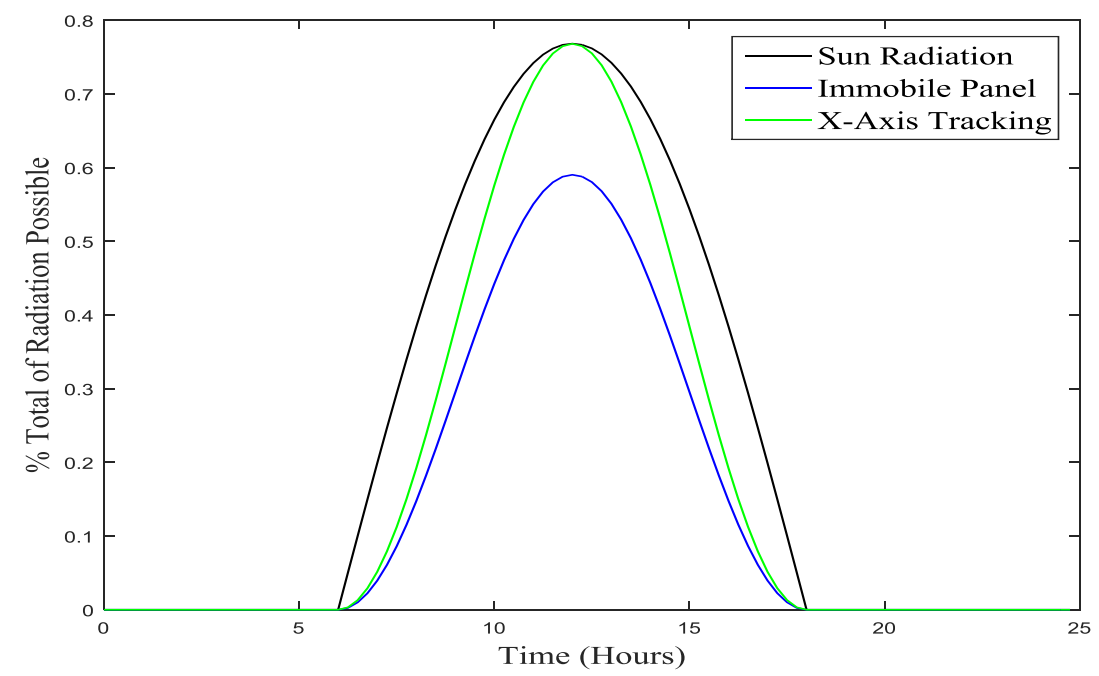

Fig.10. Absorbed solar radiation when an automatic tracker is employed in comparison to an immobile panel.

\section{CONCLUSION}

A Prototype Automatic Solar Panel Controller system was designed, constructed and tested. The device was found to track the sun as the sun radiation falling on two LDRs sensor varies. Our proposed system provides a user-friendly solution to sun tracking system. It includes several features for the comfort of the user. The provision of a hibernation mode prevents the controller from continuous monitoring of the sun location, thereby conserving over $50 \%$ of the systems energy. Future work include improving the computer interface software to be able to control more than one panel, creating a database linked to the interface software to keep record of voltage, current, charging rate of the battery at different time, and connecting the computer interface unit through a wireless link to the system, hence creating a remote access to the tracker.

\section{REFERENCES}

[1] A. R. L. Khan M. F, "Automatic sun tracking system (ASTS)," Air University, Islamabad, Pakistan., 2005.

[2] A. Kassem and M. Hamad, "A microcontroller-based multi-function solar tracking system," 2011 IEEE Int. Syst. Conf. SysCon 2011 - Proc., pp. 13-16, 2011.

[3] A. W. O. Guo Liping, Jingbo Han, "Design and simulation of a sun tracking solar power system," in 120th ASEE Annual Conference and Exposition.

[4] R. Kansal, "PIC based automatic Solar Radiation Tracker," THAPAR UNIVERSITY, Patiala, Punjab 147004, India, 2008.

[5] "Time operated solar tracking system," 2010. [Online]. Available: http://seminarprojects.com/Thread-timeoperated-. [Accessed: 07-Oct-2014].

[6] B. Lane, "Solar tracker," Cleveland State University, Cleveland, Ohio, 2008.

[7] S. A. Kalogirou, "Design and construction of a one-axis sun-tracking system," Sol. Energy, vol. 57, no. 6, pp. 465469, 1996.

[8] I. Sefa, M. Demirtas, and I. Çolak, "Application of oneaxis sun tracking system," Energy Convers. Manag., vol.
50, pp. 2709-2718, 2009.

[9] A. Kulkarni, T. Kshirsagar, A. Laturia, and P. H. Ghare, "An Intelligent Solar Tracker for Photovoltaic Panels," 2013 Texas Instruments India Educ. Conf., pp. 390-393, 2013.

[10] A. Ponniran, A. Hashim, and H. Ali Munir, "A design of single axis sun tracking system," 2011 5th Int. Power Eng. Optim. Conf. PEOCO 2011 - Progr. Abstr., no. June, pp. 107-110, 2011.

[11] A. B. Afarulrazi, W. M. Utomo, K. L. Liew, and M. Zarafi, "Solar tracker robot using microcontroller," ICBEIA 2011 - 2011 Int. Conf. Business, Eng. Ind. Appl., pp. 47-50, 2011.

[12] V. V. More, V. P., \& Kulkarni, "Design and Implementation of Microcontroller Based Automatic Solar Radiation Tracker," Int. J. Curr. Eng. Technol., no. 3, pp. 230-234, 2014.

[13] F. Duarte, P. D. Gaspar, and L. C. Gonçalves, "Two axis solar tracker based on solar maps, controlled by a lowpower microcontroller Key words 2 . Solar Maps Study and Their Application," Power Qual., vol. 5, pp. 671-676, 2010.

[14] P. Miermeister and A. Pott, "New Trends in Mechanism Science," pp. 353-360, 2010.

[15] S. S. Olokede, M. F. Ain, U. Ullah, A. Marzuki, J. J. Mohammed, S. Sreekantan, S. D. Hutagalung, Z. a Ahmad, and M. Z. Abdullah, "Feed Coupling Comparative Assessment of Selected Microstrip Patch Antenna," 8th Int. Conf. Robot. Vision, Signal Process. Power Appl., vol. 291, pp. 463-472, 2014.

[16] S. Yilmaz, H. Riza Ozcalik, O. Dogmus, F. Dincer, O. Akgol, and M. Karaaslan, "Design of two axes sun tracking controller with analytically solar radiation calculations," Renew. Sustain. Energy Rev., vol. 43, pp. 997-1005, 2015.

[17] C.-Y. Lee, P.-C. Chou, C.-M. Chiang, and C.-F. Lin, "Sun Tracking Systems: A Review," Sensors, vol. 9, pp. 38753890, 2009.

[18] H. Mousazadeh, A. Keyhani, A. Javadi, H. Mobli, K. Abrinia, and A. Sharifi, "A review of principle and suntracking methods for maximizing solar systems output," Renew. Sustain. Energy Rev., vol. 13, pp. 1800-1818, 2009.

[19] R. Dhanabal, V. Bharathi, R. Ranjitha, a. Ponni, S. 
Deepthi, and P. Mageshkannan, "Comparison of efficiencies of solar tracker systems with static panel single-axis tracking system and dual-axis tracking system with fixed mount," Int. J. Eng. Technol., vol. 5, no. 2, pp. 1925-1933, 2013.

[20] S. Deepthi, A. Ponni, R. Ranjitha, and R. Dhanabal, "Comparison of Efficiencies of Single-Axis Tracking System and Dual-Axis Tracking System with Fixed Mount,"vol. 2, no. 2, pp. 425-430, 2013.

[21] A. Samanta, A. Dutta, and S. Neogi, "A simple and efficient sun tracking mechanism using programmable logic controller," Appl. Sol. Energy, vol. 48, no. 3, pp. 218-227, 2012.

[22] M. M. Sabir and T. Ali, "Optimal PID controller design through swarm intelligence algorithms for sun tracking system," Appl. Math. Comput., vol. 274, pp. 690-699, 2016.

[23] I. J. I. Systems, M. T. Makhloufi, M. S. Khireddine, Y. Abdessemed, a Boutarfa, and B. Road, "Tracking Power Photovoltaic System using Artificial Neural Network Control Strategy," no. November, pp. 17-26, 2014.

[24] S. Chak, "A Novel Design of Spacecraft Combined Attitude \& Sun Tracking System using a Versatile Fuzzy Controller," 2015.

[25] B. M. Hamed and M. S. El-Moghany, "Fuzzy Controller Design using FPGA for Sun Tracking in Solar Array System," Int. J. Intell. Syst. Appl., vol. 4, no. February, pp. 46-52, 2012.

[26] G. Lazariou, M. Longo, M. Roscia, and M. Pagano, "Comparative analysis of fixed and sun tracking low power PV systems considering energy consumption," Energy Convers. Manag., vol. 92, pp. 143-148, 2015.

[27] Solar Choice, "Solar Trackers," 2010. [Online]. Available: http://www.solarchoice.net.au/blog/solar-trackers/. [Accessed: 16-Oct-2014].

[28] S. Aliyu, "Microcontroller based solar panel controller," Federal University of Technology, Minna, Niger St. Nigeria, 2010.

[29] Solarbotics, "Industrial Circuits Application Note Stepper Motor Basics," 2014. [Online]. Available: www.solarbotics.net/library/pd ib/pdf/motorbas.pdf. [Accessed: 07-Oct-2014].

[30] Chinmayananda D., "Monitor and control of green house environment," Dhaneswar Rath Institute of Engineering and Management Studies, Cuttack, 2010.

[31] ATMEL, "AT89C52: 8-Bit Microcontroller with 8K Bytes Flash," 2014. [Online]. Available: http://pdf1.alldatasheet.com/datasheet-. [Accessed: 07Oct-2014].

\section{Authors' Profiles}

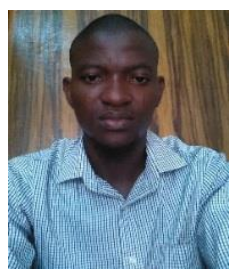

Salihu O. Aliyu is currently an Assistant Lecturer at the Department of Telecommunication Engineering, Federal University of Technology, Minna, Niger State, Nigeria. He obtained a First Class honor, BEng. Electrical and Computer Engineering at Federal University of Technology, Minna, Nigeria in 2011, after which he proceed to King Fahd University of Petroleum and Minerals, Dhahran, Saudi Arabia where he obtained his MSc in Electrical Engineering. His research is focused on Digital Signal/Image processing, Embedded System Design, Artificial Intelligence, Pattern Recognition using machine learning algorithm and Smart Grid.

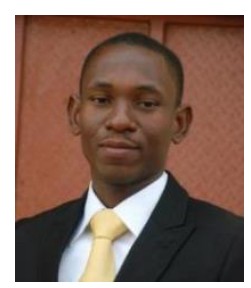

Michael Okwori is currently an Assistant Lecturer at the Department of Telecommunications Engineering, Federal University of Technology, Minna, Nigeria where he just obtained his Masters of Engineering Degree in Communications Engineering. He obtained a First Class Honors Bachelor of Engineering Degree from the Department of Electrical and Computer Engineering, Federal University of Technology Minna in 2007. His Research interests include Mobility Management in IP Networks, Mobile Telecommunication Architecture, Optical Communications, Design and Development of Smart Systems and Wireless Sensor Networks.

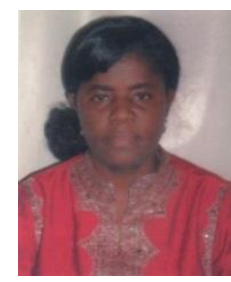

Elizabeth N. Onwuka obtained a Bachelor of Engineering (B.Eng.) Degree from Electrical and Computer Engineering Department, Federal University of Technology (FUT) Minna, Niger State, Nigeria, in October 1992; a Master of Engineering (M.Eng.) Degree, in Telecommunications, from Electrical and Computer Engineering Department, FUT, Minna, Niger State, Nigeria, in March 1998; and Doctor of Philosophy (PhD) Degree, in Communications and Information Systems Engineering, from Tsinghua University, Beijing, People's Republic of China, in June 2004. She is currently a Professor in the Department of Telecommunications Engineering FUT, Minna, Niger state. Her research interest includes Mobile communications network architecture, IP networks, handoff management, paging, network integration, and resource management in wireless networks, wireless sensor networks.

How to cite this paper: Salihu O. Aliyu, Michael Okwori, Elizabeth N. Onwuka, "A Prototype Automatic Solar Panel Controller (ASPC) with Night-time Hibernation", International Journal of Intelligent Systems and Applications (IJISA), Vol.8, No.8, pp.18-25, 2016. DOI: 10.5815/ijisa.2016.08.03 